\title{
Scale-resolved phase coherence analysis of hemispheric sunspot activity: a new look at the north-south asymmetry
}

\author{
R. Donner ${ }^{1,3}$ and M. Thiel ${ }^{2,3}$ \\ 1 Institute for Transport and Economics, Dresden University of Technology, Andreas-Schubert-Str. 23, 01062 Dresden, Germany \\ e-mail: donner@vwi.tu-dresden.de \\ 2 Department of Physics, University of Aberdeen, Aberdeen AB24 3UE, Scotland, UK \\ 3 Nonlinear Dynamics Group, Department of Physics, University of Potsdam, Am Neuen Palais 10, 14469 Potsdam, Germany
}

Received 14 September 2007 / Accepted 11 October 2007

\section{ABSTRACT}

\begin{abstract}
Aims. The sunspot activity on both solar hemispheres is known to show time intervals of different phase relationships. In this letter, we introduce an advanced statistical framework that allows an appropriate analysis of these relationships.

Methods. We combine wavelet analysis with statistical measures of phase coherence to obtain well-defined phase differences in dependence on the selected oscillation period.

Results. Our approach characterises the temporally varying phase difference of the hemispheric sunspot activity over the past 130 years, which is a major contribution to the north-south asymmetry. Coherent phase variables are found to exist for a frequency band with periodicities around the dominating 11-year cycle. We observe significant continuous long-term variability of interhemispheric phase shifts, which is not reproduced by solar activity records from previous centuries.
\end{abstract}

Key words. Sun: sunspots - Sun: activity

\section{Introduction}

The north-south asymmetry of solar activity is an intensively studied phenomenon that can be observed in terms of several indicators like the number of solar flares, the flare index, the photospheric magnetic flux, and the sunspot numbers and areas (Ballester et al. 2005). Among all these quantities, the sunspot features are probably the most studied properties of all. Continuous time series of monthly averages for sunspot numbers and areas on both solar hemispheres cover more than 130 years (from $1874 \mathrm{AD}$ to the present) and are publicly available at http://solarscience.msfc.nasa.gov/greenwch. shtml.

Classical studies of the hemispheric asymmetry of sunspot areas have mainly considered two measures: the absolute asymmetry of the sunspot areas $A_{\mathrm{n}}$ and $A_{\mathrm{s}}$ on both hemispheres,

$A A=A_{\mathrm{n}}-A_{\mathrm{s}}$

and its normalised variant,

$N A=\frac{A_{\mathrm{n}}-A_{\mathrm{s}}}{A_{\mathrm{n}}+A_{\mathrm{s}}}$

During the past decades, there has been some debate about the possible strengths and weaknesses of both approaches. In particular, both Fourier and wavelet analysis techniques have been used to find statistically significant periodicities in both indices; see Carbonell et al. (1993), Knaack et al. (2004), and Ballester et al. (2005). In addition, it has been shown that sunspot time series are usually multifractal (Carbonell et al. 1993; Sadegh Movahed et al. 2006), which further complicates their statistical analysis and interpretation.

As a new approach to quantifying the north-south sunspot asymmetry, Zolotova \& Ponyavin (2006) have recently proposed to study the phase difference between the activity on both hemispheres by means of cross-recurrence plots. Despite their claim, their method is not exclusively sensitive with respect to the phases of the respective time series, as cross-recurrence plots are known to also depend on the amplitudes (Marwan et al. 2007). As an appropriate alternative, we suggest combining wavelet analysis with statistical measures, which are used to study phase synchronisation phenomena. We demonstrate that the availability of a coherent phase definition in the case of monthly sunspot data crucially depends on the considered time scale. Such phases are only found on time scales around the 11-year sunspot cycle and are most pronounced at a reference period of 10.75 years. The resulting phase-shift dynamics are discussed as a novel measure for the north-south asymmetry and its statistical significance.

\section{Frequency-dependent phase dynamics}

Following the rising interest in the study of phase synchronisation phenomena in natural, technological, and socio-economic systems (Pikovsky et al. 2001), a variety of different approaches have been discussed for deriving meaningful phase variables from univariate time series. If there is a coherent oscillation without substantial stochastic components, the analytical signal approach is most common. This method uses the Hilbert transform to derive complex-valued signals, so that a phase variable can be defined easily. In some cases where the coherence condition is not fulfilled, this method may still be used if the difference-filtered time series is considered instead of the original record (Chen et al. 2001; Osipov et al. 2003). However, when there are noisy non-coherent oscillations on a broad frequency band, the analytic signal approach fails. 

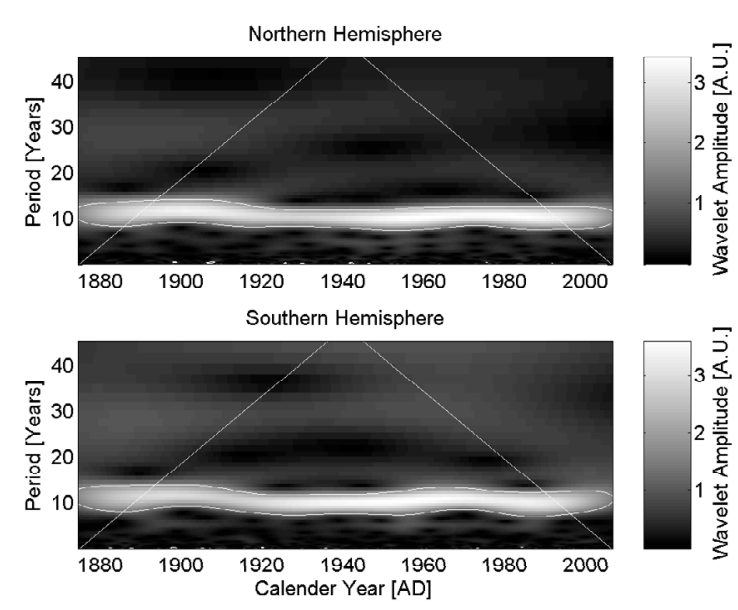

Fig. 1. Wavelet amplitudes for the northern (upper panel) and southern (lower panel) hemispheric sunspot areas. Diagonal lines indicate the cones of influence outside which no statistically significant results may be obtained. The other white lines indicate areas where the recorded variability has passed a test against red noise at a $95 \%$ probability level.

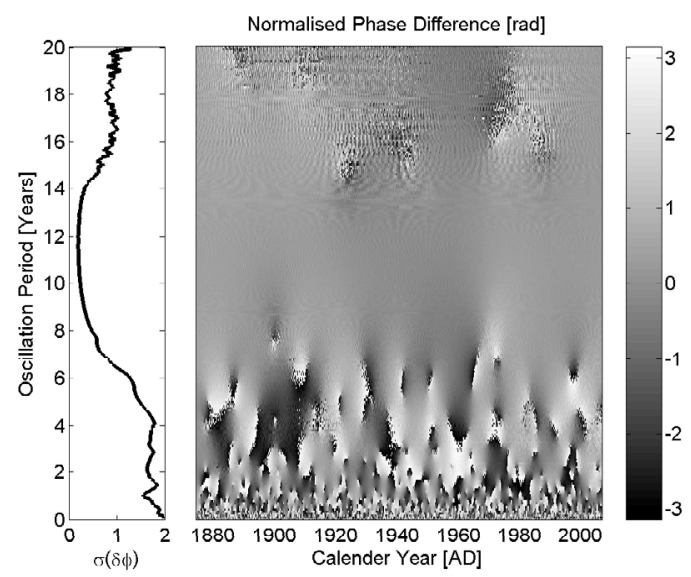

Fig. 2. Left panel: standard deviations of the phase differences over the entire time series on different reference frequencies. Right panel: phase differences $\delta \phi$ between northern and southern hemispheric sunspot activity as a function of time and reference frequency.

As an alternatve, wavelet analysis is an appropriate concept for investigating phase dynamics. In this case, only selected frequencies are considered instead of oscillations on a broad spectrum (Allefeld \& Kurths 2004; Koronovskii \& Hramov 2004; Hramov \& Koronovskii 2004). In addition, wavelet analysis has the advantage of being applicable to non-stationary systems where the analytical signal approach is found to fail. For our analysis of the hemispheric sunspot dynamics, we have therefore applied a continuous wavelet transform with a complex Morlet wavelet to decompose the time series into components that vary on different frequencies (Holschneider 1995; Grinsted et al. 2004). As shown in Fig. 1, the largest amplitudes of variability are found around the major 11-year activity cycle of the Sun for both hemispheres as expected.

For all contributions with periodicities between 1 and 240 months, the resulting phase differences between northern and southern sunspot activity were normalised to the interval $[-\pi, \pi]$. The results shown in Fig. 2 clearly indicate that these phase differences are only coherent within a narrow range of frequencies, which corresponds to time scales of about 8 to 14 years. On both smaller and on larger scales, there are no regular oscillatory
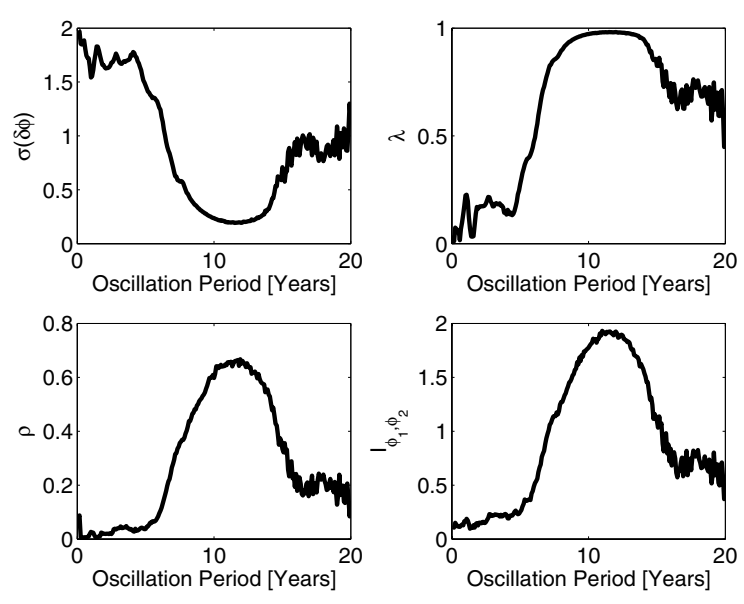

Fig. 3. Dependence of the phase coherence on the considered frequency, measured in terms of different statistical features: standard deviation of the phase differences $\sigma(\delta \phi)$, mean resultant length $\lambda$, normalised Shannon entropy $\rho$, and cross-mutual information coefficient between the phase variables $I_{\phi_{1}, \phi_{2}}$.

patterns in the corresponding frequency bands; hence, the availability of a physically meaningful phase definition depends crucially on the appropriate choice of the reference frequency.

To further quantify the coherence of the frequencydependent phases, we computed different measures that may be used as indicators of phase synchronisation between coupled oscillatory systems. Note that, in the case of the hemispheric sunspot activity, we cannot necessarily assume the existence of two coupled, self-sustained oscillators, such that the notion of synchronisation is not useful. However, the indices themselves remain meaningful because they quantify the phase coherence between the variability of the two considered records. In particular, we have considered the following measures:

- the standard deviation of normalised phase differences $\sigma(\delta \phi)$;

- the mean resultant length $\lambda$ (i.e., the "centre of gravity" of the phase differences within the unit circle), a traditional measure of directional statistics (Lord Rayleigh 1880);

- the normalised Shannon entropy $\rho$ of the relative frequencies of normalised phase differences in $M=20$ mutually disjoint intervals (Tass et al. 1998); and

- the cross-mutual information coefficient $I_{\phi_{1}, \phi_{2}}$ between the normalised phases of both time series (Paluš 1997).

The behaviour of all these indices shown in Fig. 3 demonstrates that phase coherence is found exclusively on time scales of about 8 to 14 years.

\section{Results}

According to the above results, considerations of very small or very large reference time scales leads to non-coherent behaviour of the phase variables assigned to the hemispheric sunspot activity on the respective scales. In the following, we have therefore focussed our attention on periodicities in the coherent range around 11 years as the reference time scales. As all noisy highfrequency components have been filtered out in this case, the resulting phases are well-defined and may be used to study the varying relationship between northern and southern hemispheric sunspot activity. As long as the considered scales are below (above) the mean periodicity of the sunspot cycle over the considered time interval, these phases show an average drift towards 

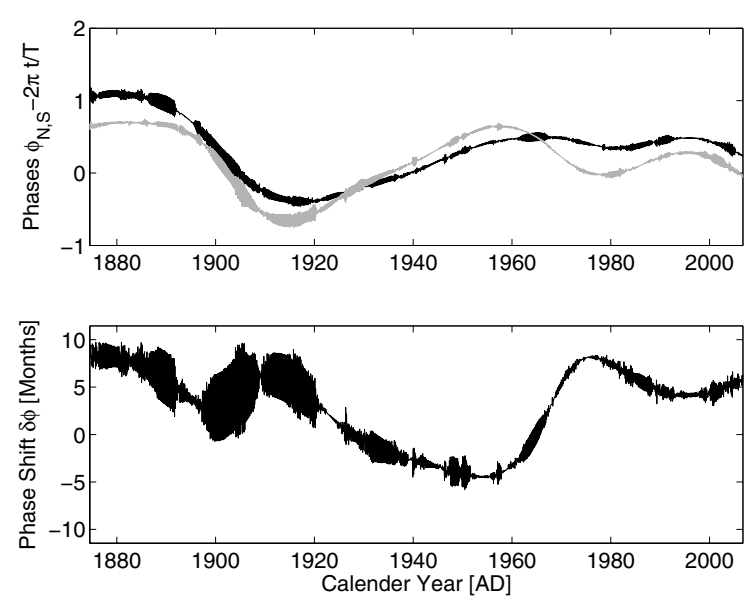

Fig. 4. Upper panel: phases of northern and southern hemispheric sunspot activity $\phi_{\mathrm{N}}$ (black) and $\phi_{\mathrm{S}}$ (gray) after substracting a linearly increasing phase signal with a periodicity of $T=10.75$ years, which represents the average duration of a sunspot cycle during the past 130 years. Lower panel: the resulting phase difference $\delta \phi$ between the activity on both hemispheres.

negative (positive) values with respect to the respective reference frequencies. The time scale for which this drift is minimised may thus be identified with the underlying basic periodicity. In our considerations, the optimum has been found for $T=10.75$ years, which matches recent estimates of the average sunspot cycle frequency reasonably well (Frick et al. 1997; Faria et al. 2004).

Figure 4 shows that the drift of the phases of the sunspot areas differs between both hemispheres. As a result, the interhemispheric phase difference shows continuous transitions from those conditions where the variability in the northern hemisphere occurs earlier than in the southern hemisphere to those where the opposite is true, and returning back again, all of this on long time scales. The maximum observed phase shift is below \pm 10 months, which means less than $10 \%$ of the observed average cycle period of 10.75 years. Hence, there is indeed a strong coherence between the dynamics of both hemispheres on this time scale, but with a variable delay. The corresponding variability pattern of $\delta \phi$ is robust against changes in the reference frequency within a reasonable range.

It has to be pointed out that similar variations in phase differences are often found in phase synchronised chaotic or noisy oscillatory systems. In these cases, the corresponding phenomenon can be attributed to the phase diffusion of the oscillators (Pikovsky et al. 2001). As the hemispheric sunspot activity probably represents two observables of the same complex system, one should speak not of phase synchronisation but rather of phase coherence or a joint drift of the phases. One possible explanation for this behaviour would be that the sunspot activity is governed by a single process for both hemispheres. The observations of southern and northern sunspot activity reflect that behaviour, but have random fluctuations. However, two different, but coupled processes for north and south would also be consistent with the observations.

To prove the statistical significance of the derived phase pattern, we compared the phase differences to those taken with respect to a signal that is as close as possible related to the hemispheric sunspot areas. For this approach, we chose the monthly values of international sunspot numbers from earlier recordings. Corresponding time series starting in 1749 are available at
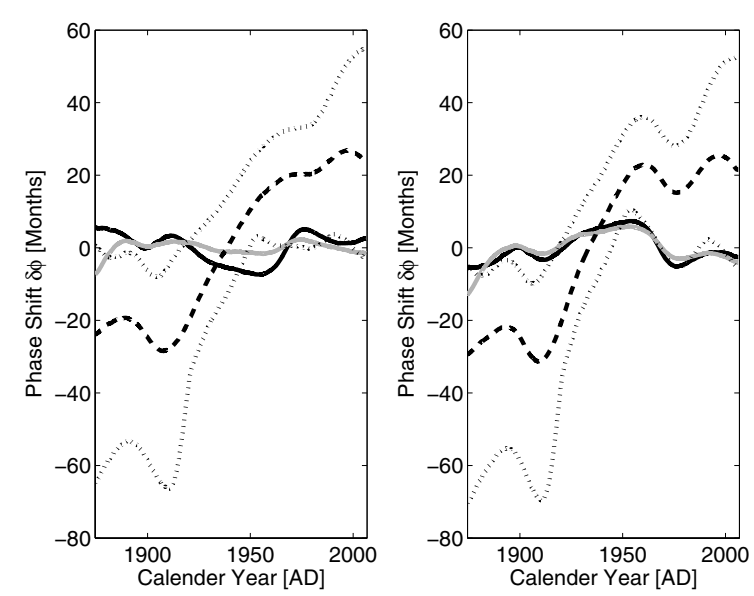

Fig. 5. Phase differences of the hemispheric sunspot areas with respect to the areas on the other hemisphere (solid black lines), the international sunspot number for the reference period (solid gray lines), and the natural surrogates for the northern (left panel) and southern (right panel) hemisphere. Dashed and dotted lines indicate the average values and the $5 \%$ and $95 \%$ probability quantiles from the distribution of phase differences with respect to the surrogates, respectively. All time series have been smoothed by a centred moving-average filter with a width of two years.

WWW.ngdc.noaa.gov/stp/SOLAR/ftpsunspotnumber.html and are possibly the longest continuous (gap-free) record of solar activity. The phase behaviour of the hemispheric sunspot areas and the total sunspot numbers over the past 130 years is dynamically rather similar (apart from a different phase diffusion). However, these "natural surrogates" allow us to statistically test whether the dynamics within this time interval differ significantly from what is observed in earlier periods (van Leeuwen et al. 2003; Thiel et al. 2006).

According to Fig. 5, there are time intervals where the phase differences between the sunspot areas and the simultaneously recorded sunspot numbers deviate significantly from those calculated with respect to sunspot numbers from earlier periods. In particular, the surrogates show a clear transition of the phase behaviour, which is very likely related to changes in the average cycle frequency over the last few centuries. Interestingly, the phases of the international sunspot number seem to coincide closely with those of the northern hemispheric sunspot areas, which can be seen from the corresponding phase differences with respect to the areas on the southern hemisphere.

The interhemispheric phase shift may be considered as an improved indicator for the north-south asymmetry of the sunspot activity. While studying the long-term variability of this indicator, we have found that there are no statistically significant periodicities of about 22 years (Swinson et al. 1986) or 44 years (Knaack et al. 2004; Ballester et al. 2005), which correspond to the magnetic cycle of the Sun and its second harmonics. Hence, it is possible that these periodicities are introduced by variations in the maximum cycle amplitudes rather than those of the respective oscillation phases. Long periodicities, such as the 110-year cycle reported by Verma (1992), cannot be resolved due to the limited length of the time series (cf. Ballester et al. 2005), but the inferred variability pattern of the hemispheric phase difference does not suggest a correspondingly regular period. Further short-term periodicities (reported by Knaack et al. 2004; Forgács-Dajka \& Borkovits 2007) cannot be resolved by our method, because the restriction to wavelet components that vary on longer time scales filters all components with higher frequencies. 


\section{Conclusions}

We have demonstrated that applying methods from phase synchronisation analysis improves our insight into the long-term dynamics of the north-south asymmetry of sunspot activity. Since the analysis of phase relationships between observables in natural systems requires a proper phase definition, such a definition may be achieved by selecting a frequency that dominates the variability pattern and allows us to define a coherent signal in the case of systems oscillating on multiple scales. As we have demonstrated, wavelet analysis is an appropriate tool for this purpose.

We used the wavelet components to study the frequencydependent phase difference of hemispheric sunspot variability over the past 130 years. Our results suggest that the coherence necessary for a proper phase definition is only present in a small frequency band with periodicities between about 8 and 14 years, which corresponds to the well-known solar activity cycle. Clear evidence for strong phase coherence is found by fixing a wavelet component within this range. In contrast to this, Zolotova \& Ponyavin (2006) have applied a moving-average filter with a width of one year, which corresponds to the minimal resolved period of oscillations. For the corresponding frequency, our results indicate that phases are not well-defined for the respective activity time series; i.e., the phase differences obtained on this scale have hardly any statistical meaning.

A detailed inspection of the derived phases shows that the average periodicity of the sunspot variations is about 10 years and 9 months. Our analysis of the variability of the phase-difference on the corresponding frequency band reveals continuous transitions from those conditions where the sunspot activity in the northern hemisphere occurs earlier than in the southern hemisphere (positive phase shifts before 1925) to those where the opposite is true (negative phase shifts between about 1925 and 1965), and returning to the earlier conditions. Our results provide detailed quantification of this variable phase shift on multidecadal time scales; however, the available data cover a time interval that is too short to draw any conclusions about eventual long-term cyclicities. After comparing Fig. 4 to earlier studies of sunspot asymmetry indices, we have to conclude that the oscillating hemispheric phase shift is probably not directly related to the dynamics of these indices. In particular, the north-south asymmetry does not appear to be the result of a "phase asynchronisation", as suggested by Zolotova \& Ponyavin (2006), but of hemispherically different phase diffusion. However, our analyses do not allow a particular physical mechanism to be assigned to the observed behaviour, which is consistent with, for example, the synchronisation of two coupled self-sustained oscillators, two independent components of the same oscillating system, or even the assumption of one system that is viewed by two different observation functions.
We suggest that the temporally varying phase relationship between the northern and southern hemispheric sunspot areas is a main contribution to the north-south asymmetry of solar activity. To validate this hypothesis further, one has to systematically compare the absolute and normalised sunspot asymmetry (Eqs. (1) and (2)) with their values for phase-adjusted time series of hemispheric activity. For this purpose, it seems to be more promising to refine the cross-recurrence approach of Zolotova \& Ponyavin (2006) even more instead of using the method proposed here, as the latter depends explicitly on the scale. Further investigations of this question have been planned for our future research.

Acknowledgements. R.D. acknowledges financial support from the Helmholtz virtual institute "Pole-Equator-Pole" (PEP) and the German Research Foundation. M.T. was supported by the DFG priority program 1114 "Mathematical methods for time series analysis and digital image processing" and by an RCUK fellowship from the EPSRC. The wavelet analysis was performed with the MATLAB wavelet toolbox and the wavelet coherence package by A. Grinsted, J. C. Moore, and S. Jevrejeva (http://www.pol.ac.uk/home/research/waveletcoherence/).

\section{References}

Allefeld, C., \& Kurths, J. 2004, Int. J. Bif. Chaos, 14, 417

Ballester, J. L., Oliver, R., \& Carbonell, M. 2005, A\&A, 431, L5

Carbonell, M., Oliver, R., \& Ballester, J. L. 1993, A\&A, 274, 497

Chen, J. Y., Wong, K. W., \& Shuai, J. W. 2001, Phys. Lett. A, 285, 312

Faria, H. H., Echer, E., Rigozo, N. R., et al. 2004, Sol. Phys., 223, 305

Forgács-Dajka, E., \& Borkovits, T. 2007, MNRAS, 374, 282

Frick, P., Galyagin, D., Hoyt, D. V., et al. 1997, A\&A, 328, 670

Grinsted, A., Moore, J. C., \& Jevrejeva, S. 2004, Nonlin. Proc. Geophys., 11, 561

Holschneider, M. 1995, Wavelets: An Analysis Tool (Oxford: Oxford Univ. Press)

Hramov, A., \& Koronovskii, A. 2004, Chaos, 14, 603

Knaack, R., Stenflo, J. O., \& Berdyugina, S. V. 2004, A\&A, 418, L17

Koronovskii, A., \& Hramov, A. 2004, Techn. Phys. Lett., 30, 29

Lord Rayleigh 1880, Phil. Mag. Ser. 5, 10, 73

Marwan, N., Romano, M. C., Thiel, M., \& Kurths, J. 2007, Phys. Rep., 438, 237

Osipov, G. V., Hu, B., Zhou, C., Ivanchenko, M. V., \& Kurths, J. 2003, Phys. Rev. Lett., 91, 024101

Paluš, M. 1997, Phys. Lett. A, 235, 341

Pikovsky, A., Rosenblum, M. G., \& Kurths, J. 2001, Synchronization (Cambridge: Cambridge Univ. Press)

Sadegh Movahed, M., Jafari, G. R., Ghasemi, F., Rahvar, S., \& Rahimi Tabar, M. R. 2006, J. Statist. Mech., 2, P02003

Swinson, D. B., Koyama, H., \& Saito, T. 1986, Sol. Phys., 106, 35

Tass, P., Rosenblum, M. G., Weule, J., et al. 1998, Phys. Rev. Lett., 81, 3291

Thiel, M., Romano, M. C., Kurths, J., Rolfs, M., \& Kliegl, R. 2006, Europhys. Lett., 75, 535

van Leeuwen, P., Geue, D., Lange, S., et al. 2003, BMC Physiol., 3, 2

Verma, V. K. 1992, in The Solar Cycle, ASP Conf. Ser., ed. K. L. Harvey, 27, 429

Zolotova, N. V., \& Ponyavin, D. I. 2006, A\&A, 449, L1 